\title{
Quantum Logic with a Single Trapped Electron
}

\author{
Stefano Mancini ${ }^{1}$, Ana M. Martins ${ }^{2}$ and Paolo Tombesi ${ }^{3}$ \\ ${ }^{1}$ Dipartimento di Fisica and Unità INFM, \\ Università di Milano, Via Celoria 16, I-20133 Milano, Italy \\ ${ }^{2}$ Centro de Fisica de Plasmas, Instituto Superior Tecnico, \\ P-1096 Lisboa Codex, Portugal \\ ${ }^{3}$ Dipartimento di Matematica e Fisica and Unità INFM, \\ Università di Camerino, I-62032 Camerino, Italy
}

(Received: July 12, 2021)

\begin{abstract}
We propose the use of a trapped electron to implement quantum logic operations. The fundamental controlled-NOT gate is shown feasible. The two quantum bits are stored in the internal and external (motional) degrees of freedom.
\end{abstract}

\section{INTRODUCTION}

The modern theory of information relies on the very foundations of quantum mechanics. This is because of information is physical, as recently emphasised by Landauer [i]. It implies that the laws of quantum mechanics can be used to process and store information. The elementary quantity of classical information is the bit, which is represented by a dichotomic system; therefore, any physical realization of a bit needs a system with two states. The very novel characteristics of quantum information is that, by using quantum states to store information, a quantum system can be in a superposition of states. This means, in a sense, that the elementary quantity of quantum information, a quantum bit, can be in both the states at the same time.

Already in 1981 Feynman [2] pointed out the impossibility for a classical computer to simulate the evolution of a quantum system in an efficient way. This opened the search of a more efficient way to simulate quantum systems until Deutsch [3] provided a satisfactory theoretical description of a universal quantum computer. The quantum computer is a device which operates with simple quantum logic gates. These are analogous to the classical gates, which perform one elementary operation on two bits in a given way. Quantum logic gates differ from their classical counterpart in that they operate on quantum superpositions and perform operations on them [4. It has also been shown that any quantum computation can be built from a series of one-bit and two-bit quantum logic gates [5]. The fundamental quantum logic gate is the controlled-NOT $(\mathrm{CN})$ gate [6.7], in which one quantum bit (or qubit) is flipped (rotated by $\pi$ radians) depending upon the state of a second qubit.

A very promising candidate for quantum logic was recently introduced by Cirac and Zoller [8], who showed how to construct universal multibit quantum logic gates in a system of laser-cooled trapped ions. Other systems were devised as building blocks for a quantum computer [9], the search for new systems is, however, still open because none of the previous systems is yet claimed as the best candidate. One should devise a system with very low loss, almost de-coherence free, which can be well controlled with simple operations. However, before obtaining a suitable system one has to be sure that the mathematical models of quantum logic could be easily implemented in a real physical system. Up to now the experimental realization of such logic operations were shown to be possible with trapped ions [10], flying qubits [11], and cavity QED [12]. There are claims that the quantum logic gates are obtained in NMR systems 13. but this was also questioned [14]. In these systems, however, the implementation of quantum logic is not at all easy and was not completely performed in all of them.

It is here our aim to show that other natural candidates to implement quantum logic could be trapped electrons. In fact, an electron is a real two-state system and when stored in a Penning trap 115 permits very accurate measurements [16]. Furthermore, in such a system the decoherence effects, which can destroy the quantum interference that enables the quantum logic implementation [17], are well controlled [18]. Moreover, electrons being structureless, open other possibilities, e.g. the use of statistics that has not as yet been considered in the literature.

To introduce the system, in this paper we consider a single electron trapped in a Penning trap, and we show how to get a controlled-NOT gate on a pair of qubits. The two qubits comprise two internal (spin) states and two external (quantized harmonic motion) states. Although this minimal system consists of only two qubits, it illustrates the basic operations necessary for, and the problems associated with, quantum logic networks with electrons. The extension to 
two o more electrons needs more investigations. Here we are not interested in the scalability of the system, rather to show the physical implementation of quantum logic in a readly controllable way with the existing technologies.

\section{THE MODEL}

We are considering the "geonium" system [18] consisting of an electron of charge $e$ and mass $m$ moving in a uniform magnetic field $\mathbf{B}$, along the positive $z$ axis, and a static quadrupole potential

$$
V=V_{0} \frac{x^{2}+y^{2}-2 z^{2}}{4 d^{2}}
$$

where $d$ characterizes the dimension of the trap and $V_{0}$ is the potential applied to the trap electrodes 18 .

In this work, in addition to the usual trapping fields, we embed the trapped electron in a radiation field of vector potential $\mathbf{A}_{\text {ext }}$. Traditional hyperbolic Penning traps form cavities for which it has not yet been possible to even classify the standing-wave fields. In marked contrast, the radiation modes of a simple cylindrical cavity are classified in a familiar way as either transverse magnetic or transverse electric modes [19,20]. So, in the following, we always refer to such cylindrical traps.

The Hamiltonian for the trapped electron can be written as the quantum counterpart of the classical Hamiltonian with the addition of the spin term

$$
H=\frac{1}{2 m}[\mathbf{p}-e \mathbf{A}]^{2}+e V-\frac{g}{2} \frac{e \hbar}{2 m} \sigma \cdot \mathbf{B}
$$

where $g$ is the electron's $g$ factor, and

$$
\mathbf{A}=\frac{1}{2} \mathbf{B} \wedge \mathbf{r}+\mathbf{A}_{\mathrm{ext}}
$$

where $\mathbf{r} \equiv(x, y, z), \mathbf{p} \equiv\left(p_{x}, p_{y}, p_{z}\right)$ are respectively the position and the conjugate momentum operators, while $\sigma \equiv\left(\sigma_{x}, \sigma_{y}, \sigma_{z}\right)$ are the Pauli matrices in the spin space.

The motion of the electron in absence of the external field $\mathbf{A}_{\text {ext }}$ is the result of the motion of three harmonic oscillators 18], the cyclotron, the axial and the magnetron, well separated in the energy scale, plus a spin precession around the $z$ axis. This can be easily understood by introducing the ladder operators

$$
\begin{aligned}
& a_{z}=\sqrt{\frac{m \omega_{z}}{2 \hbar}} z+i \sqrt{\frac{1}{2 \hbar m \omega_{z}}} p_{z} \\
& a_{c}=\frac{1}{2}\left[\sqrt{\frac{m \omega_{c}}{2 \hbar}}(x-i y)+\sqrt{\frac{2}{\hbar m \omega_{c}}}\left(p_{y}+i p_{x}\right)\right] \\
& a_{m}=\frac{1}{2}\left[\sqrt{\frac{m \omega_{c}}{2 \hbar}}(x+i y)-\sqrt{\frac{2}{\hbar m \omega_{c}}}\left(p_{y}-i p_{x}\right)\right]
\end{aligned}
$$

where the indexes $z, c$ and $m$ stand for axial, cyclotron and magnetron respectively. The above operators obey the commutation relation $\left[a_{i}, a_{j}^{\dagger}\right]=\delta_{i j}, i, j=z, c, m$.

When $\mathbf{A}_{\text {ext }}=0$, the Hamiltonian (2) simply reduces to

$$
H=\hbar \omega_{z} a_{z}^{\dagger} a_{z}+\hbar \omega_{c} a_{c}^{\dagger} a_{c}-\hbar \omega_{m} a_{m}^{\dagger} a_{m}+\frac{\hbar}{2} \omega_{s} \sigma_{z}
$$

where the angular frequencies are given by

$$
\omega_{z}=\sqrt{\frac{|e| V_{0}}{m d^{2}}} ; \quad \omega_{c}=\frac{|e| B}{m} ; \quad \omega_{m} \approx \frac{\omega_{z}^{2}}{2 \omega_{c}} .
$$

and $\omega_{s}=g|e| B / 2 m$ is the spin precession angular frequency. In the previous expression for $\omega_{c}$ we neglected very small corrections [18] which are not relevant for our purpose. In typical experimental configurations 18] the respective frequency ranges are $\omega_{z} / 2 \pi \simeq \mathrm{MHz}, \omega_{c} / 2 \pi \simeq \mathrm{GHz}$, and $\omega_{m} / 2 \pi \simeq \mathrm{kHz}$.

Let us introduce the external radiation field as a standing wave along the $z$ direction and rotating, i.e. circularly polarized, in the $x-y$ plane with frequency $\Omega$ [21]. In particular, we consider a standing wave within the cilindrical cavity with wave vector $k$ and amplitude $|\alpha|$. Then, we can write 


$$
\mathbf{A}_{\mathrm{ext}}=\left(i\left[e^{i \varphi+i \Omega t}-e^{-i \varphi-i \Omega t}\right],\left[e^{i \varphi+i \Omega t}+e^{-i \varphi-i \Omega t}\right], 0\right) \times|\alpha| \cos (k z+\phi),
$$

where $\varphi$ is the phase of the wave field which gives the direction of the electric (or magnetic) vector in the $x-y$ plane at the initial time. We assume this can be experimentally controlled. The amplitude $|\alpha|$ should depend upon the transverse spatial variables through the Bessel function [19] but we can consider it as a constant because of the small radius of the ciclotron motion [20]. The phase $\phi$ definines the position of the center of the axial motion with respect to the wave. Depending on its value the electron can be positioned in any place between a node and an antinode.

For frequencies $\Omega$ close to $\omega_{c}$ and $\omega_{s}$, we can neglect the slow magnetron motion, then the Hamiltonian (2) becomes

$$
\begin{aligned}
H & =\hbar \omega_{z} a_{z}^{\dagger} a_{z}+\hbar \omega_{c} a_{c}^{\dagger} a_{c}+\frac{\hbar}{2} \omega_{s} \sigma_{z} \\
& +\hbar \epsilon\left[a_{c} e^{i \varphi+i \Omega t}+a_{c}^{\dagger} e^{-i \varphi-i \Omega t}\right] \cos (k \hat{z}+\phi) \\
& +\hbar \zeta\left[\sigma_{-} e^{i \varphi+i \Omega t}+\sigma_{+} e^{-i \varphi-i \Omega t}\right] \sin (k \hat{z}+\phi),
\end{aligned}
$$

where

$$
\epsilon=\left(\frac{2|e|^{3} B}{\hbar m^{2}}\right)^{1 / 2}|\alpha|, \quad \zeta=\frac{g|e|}{2 m}|\alpha| k,
$$

and $\sigma_{ \pm}=\left(\sigma_{x} \pm i \sigma_{y}\right) / 2$. The fourth and fifth terms in the right hand side of the Hamiltonian (10) describe the interaction between the trapped electron and the standing wave which can give rise to a coupling between the axial and cyclotron motions, as well as between the axial and spin ones. In writing Eq. (10) we omitted terms coming from $\mathbf{A}_{\text {ext }}^{2}$ which give a negligible contribution (at most an axial frequency correction) when the electron in positioned in a node or antinode as we shall do in the following.

\section{ENTANGLED STATES PREPARATION}

The spin state is usually controlled through a small oscillatory magnetic field $\mathbf{b}$ that lies in the $x-y$ plane 18

$$
\mathbf{b}(t)=b\left(\cos \left(\omega_{s} t+\theta\right), \sin \left(\omega_{s} t+\theta\right), 0\right),
$$

which causes Rabi oscillations at frequency $\varpi_{s}=g|e| b / 2 \mathrm{~m}$. The phase $\theta$ can be experimentally controlled; it gives the direction of the field at initial times. The Hamiltonian that follows from Eq. (12), in absence of the standing wave and in a frame rotating at frequency $\omega_{s}$, is

$$
H_{s}=\hbar \frac{\varpi_{s}}{2}\left[\sigma_{+} e^{-i \theta}+\sigma_{-} e^{i \theta}\right]=\hbar \frac{\varpi_{s}}{2}\left[\sigma_{x} \cos \theta+\sigma_{y} \sin \theta\right] .
$$

The other non interacting terms do not affect the spin motion and can be neglected. The evolution of the spin state $|\chi\rangle_{s}=u|\uparrow\rangle+v|\downarrow\rangle$, with $|u|^{2}+|v|^{2}=1$, under such Hamiltonian will be

$$
|\chi(t)\rangle_{s}=\left[u \cos \left(\frac{\varpi_{s} t}{2}\right)-i v e^{-i \theta} \sin \left(\frac{\varpi_{s} t}{2}\right)\right]|\uparrow\rangle+\left[v \cos \left(\frac{\varpi_{s} t}{2}\right)-i u e^{i \theta} \sin \left(\frac{\varpi_{s} t}{2}\right)\right]|\downarrow\rangle .
$$

Thus, depending on the interaction time, any superposition of spin states can be generated.

For what concerns the spatial degrees of freedom, we assume the cyclotron and the axial motions are deep cooled down to their respective lower states, i.e. $|0\rangle_{c}$ and $|0\rangle_{z}$. This could be achievable when the axial motion is decoupled from the external circuit usually used to extract information [18,20].

We now consider the spin and the axial degrees of freedom as qubits. Then, by choosing $\phi=0$, i.e. positioning the electron in the node of the standing wave, Eq. (10) can be approximated by

$$
\begin{aligned}
H & =\hbar \omega_{z} a_{z}^{\dagger} a_{z}+\hbar \omega_{c} a_{c}^{\dagger} a_{c}+\frac{\hbar}{2} \omega_{s} \sigma_{z} \\
& +\hbar \epsilon\left[a_{c} e^{i \varphi+i \Omega t}+a_{c}^{\dagger} e^{-i \varphi-i \Omega t}\right] \\
& +\hbar \zeta k \sqrt{\frac{\hbar}{2 m \omega_{z}}}\left[\sigma_{-} e^{i \varphi+i \Omega t}+\sigma_{+} e^{-i \varphi-i \Omega t}\right]\left(a_{z}+a_{z}^{\dagger}\right) .
\end{aligned}
$$


We distinguish two situations (in a frame rotating at frequency $\Omega$ ): the first one in which $\Omega=\omega_{s}-\omega_{z}$ gives

$$
H_{-}=\hbar \eta\left[\sigma_{+} a_{z} e^{-i \varphi}+\sigma_{-} a_{z}^{\dagger} e^{i \varphi}\right]
$$

where $\eta=k \zeta \sqrt{\hbar / 2 m \omega_{z}}$.

The second, for which $\Omega=\omega_{s}+\omega_{z}$ gives

$$
H_{+}=\hbar \eta\left[\sigma_{+} a_{z}^{\dagger} e^{-i \varphi}+\sigma_{-} a_{z} e^{i \varphi}\right] .
$$

The action of Hamiltonian (16) for a time $t$ over an initial state $|0\rangle_{z}|\uparrow\rangle$ leads to

$$
|0\rangle_{z}|\uparrow\rangle \rightarrow \cos (\eta t)|0\rangle_{z}|\uparrow\rangle-i e^{i \varphi} \sin (\eta t)|1\rangle_{z}|\downarrow\rangle .
$$

Instead, the action of Hamiltonian (17) for a time $t$ over an initial state $|0\rangle_{z}|\downarrow\rangle$ leads to

$$
|0\rangle_{z}|\downarrow\rangle \rightarrow \cos (\eta t)|0\rangle_{z}|\downarrow\rangle-i e^{-i \varphi} \sin (\eta t)|1\rangle_{z}|\uparrow\rangle .
$$

Practically, if the electron enters in the trap with e.g. its spin down, by applying selectively the Hamiltonians (13), (16) and (17) for appropriate times we can get states of the form

$$
\alpha|0\rangle_{z}|\downarrow\rangle+\beta|0\rangle_{z}|\uparrow\rangle+\gamma|1\rangle_{z}|\downarrow\rangle+\delta|1\rangle_{z}|\uparrow\rangle, \quad|\alpha|^{2}+|\beta|^{2}+|\gamma|^{2}+|\delta|^{2}=1,
$$

which show entanglement between the two qubits.

Therefore, the manipulation between the four basis eigenstates spanning the two-qubit register $\mathcal{B} \equiv\left\{|0\rangle_{z} \mid \downarrow\right.$ \rangle$\left.,|0\rangle_{z}|\uparrow\rangle,|1\rangle_{z}|\downarrow\rangle,|1\rangle_{z}|\uparrow\rangle\right\}$ is achievable.

\section{LOGIC OPERATIONS}

Here we shall consider the spin as "target" qubit, and the axial degree as "control" qubit. The basic logic operations on a single qubit (e.g. Hadamard gate) can be implemented in the target qubit by applying the Hamiltonian (13), while there is no way to control directly the axial qubit.

The CN gate represents, instead, a computation at the most fundamental level: the target qubit is flipped depending upon the state of the control qubit.

The truth table of the reduced $\mathrm{CN}$ gate is

$$
\begin{aligned}
|0\rangle_{z}|\downarrow\rangle & \rightarrow|0\rangle_{z}|\downarrow\rangle, \\
|0\rangle_{z}|\uparrow\rangle & \rightarrow|0\rangle_{z}|\uparrow\rangle, \\
|1\rangle_{z}|\downarrow\rangle & \rightarrow|1\rangle_{z}|\uparrow\rangle, \\
|1\rangle_{z}|\uparrow\rangle & \rightarrow|1\rangle_{z}|\downarrow\rangle .
\end{aligned}
$$

To implement such a transformation we consider $\Omega=\omega_{s}$ and $\phi=-\pi / 2$, i.e. the electron is positioned in an antinode (this operation is routinely performed in actual experiments [20]). Then, the leading term of Eq. (10) (in a frame rotating at frequency $\Omega$ ) will result

$$
H=-\hbar \zeta\left[\sigma_{+} e^{-i \varphi}+\sigma_{-} e^{i \varphi}\right] \times\left[1-\frac{\hbar k^{2}}{4 m \omega_{z}}-\frac{\hbar k^{2}}{2 m \omega_{z}} a_{z}^{\dagger} a_{z}\right]
$$

If we choose $\varphi=0$, the above Hamiltonian reduces to

$$
H=-\hbar 2 \zeta\left(1-\frac{\hbar k^{2}}{4 m \omega_{z}}\right) \sigma_{x}+\hbar 2 \zeta \frac{\hbar k^{2}}{2 m \omega_{z}} a_{z}^{\dagger} a_{z} \sigma_{x} .
$$

Of course, for logic operations on the two qubits, only the interacting part of the above Hamiltonian is relevant. On the other hand the flipping effect of the first term of Hamiltonian (23) can be eliminated by a successive action of Hamiltonian (13) with $\theta=0$, for a time $\tau$ such that

$$
\tau \varpi_{s}=4 \zeta\left(1-\frac{\hbar k^{2}}{4 m \omega_{z}}\right) t^{*} \pm 2 \pi n
$$


where $n$ is a natural number and $t^{*}$ is the interaction time with Hamiltonian (23).

Hence, the relevant Hamiltonian for the $\mathrm{CN}$ gate is

$$
H=\hbar \kappa a_{z}^{\dagger} a_{z} \sigma_{x}
$$

where $\kappa=\hbar \zeta k^{2} / m \omega_{z}$.

If we appropriately choose the interaction time $t^{*}=\pi / 2 \kappa$ we can apply the transformation

$$
U=\exp \left(-i \pi a_{z}^{\dagger} a_{z} \sigma_{x} / 2\right) .
$$

Thus, the net unitary transformation, in the $\mathcal{B}$ basis, is

$$
\left(\begin{array}{c}
1,0,0,0 \\
0,1,0,0 \\
0,0,0,-i \\
0,0,-i, 0
\end{array}\right) .
$$

This transformation is equivalent to the reduced $\mathrm{CN}$ gate of Eq. (21), apart from phase factors that can be eliminated by the appropriate phase settings of subsequent logic operations [7]. Practically, the reduced CN gate consists here in a single step similarly to Ref. [22].

\section{INFORMATION MEASUREMENTS}

We recall that in the geonium system the measurements are performed on the axial degree of freedom due to the nonexistence of good detectors in the microwave regime [18]. The oscillating charged particle induces alternating image charges on the electrodes, which in turn cause an oscillating current to flow through an external circuit where the measurement is performed. The current will be proportional to the axial momentum $p_{z}$ [18]. The very act of measurement changes, however, the state of the measured observable. Then, in order not to loose any stored information because of the measurement, we shall transfer the information contained in the axial qubit into the cyclotron degree of freedom prior to the measurement procedure. This will allow us to get a complete information about the qubits by coupling different cyclotron and spin observables with the axial degree of freedom.

To transfer the information from the axial motion to the cyclotron one, we again use the standing wave, but with another resonance, $\Omega=\omega_{c}-\omega_{z}$ in order to get from Eq. (10)

$$
H=i \hbar \epsilon k \sqrt{\frac{\hbar}{2 m \omega_{z}}}\left(a_{c}^{\dagger} a_{z}-a_{c} a_{z}^{\dagger}\right) .
$$

Here we set $\phi=\varphi=-\pi / 2$. With the action of the Hamiltonian (28) for a well chosen interaction time, it is possible to transfer any previously entangled state as follows

$$
|0\rangle_{c}\left[c_{0}|0\rangle_{z}|\chi\rangle_{s}+c_{1}|1\rangle_{z}\left|\chi^{\prime}\right\rangle_{s}\right] \rightarrow\left[c_{0}|0\rangle_{c}|\chi\rangle_{s}+c_{1}|1\rangle_{c}\left|\chi^{\prime}\right\rangle_{s}\right]|0\rangle_{z},
$$

where $|\chi\rangle$ and $\left|\chi^{\prime}\right\rangle$ represent two generic spin states. This is obtained when the interaction time is $t=\sqrt{\pi m \omega_{z} / 2 \hbar \epsilon k}$.

Once the information is transferred to the cyclotron degree of freedom, the axial motion is coupled with the external circuit, and it will reach the thermal equilibrium with the read-out apparatus.

Then, the measurements of $a_{c}^{\dagger} a_{c}$ and $\sigma_{z}$ can be done in the usaul way with the aid of the magnetic bottle which causes a shift of the axial resonance proportional to the respective quantum numbers [18]

$$
\Delta \omega_{z} \approx \tilde{\omega}_{z}\left(\frac{g s}{4}+n_{c}+\frac{1}{2}\right)
$$

where $\tilde{\omega}_{z}$ is a constant, and $n_{c}, s$ are the cyclotron excitacion and spin quantum numbers. This frequency shift can be measured with very high precision $[18]$.

In this model it could be also possible to obtain phase information about the quantum state of the register by means of the coupling between the meter (axial degree) and the system (cyclotron or spin) induced again by the standing waves (see e.g. Ref. [21]). 


\section{CONCLUSIONS}

In conclusion, we have shown the possibility of using a trapped electron for fundamental quantum logic. That system has the advantage of a well defined and simple internal structure and, practically, the decoherence appears only in the axial degree of freedom as a consequence of measurements but the information stored in this degree of freedom, prior to the measurement, can be unitarily transferred into the cyclotron motion. The latter can be preserved from decoherence due to decay mechanisms by appropriately tuning the cavity 24 . The spin is very stable against fields fluctuations [25]. Eventually, the register $\mathcal{B}$, in such a configuration, could only suffer of the time uncertainty in the switching on and off the interactions, possibly leading to nondissipative decoherence [26,27]. The effect on the fidelity in performing the logical operations could arise, indeed, from the impurity of the motional ground states due to an imperfect cooling process. Anyway, we retain that the present model can be implemented with the current technology, and a comparison with the results obtained in the experiment of Ref. [28] would be useful. With respect to the last Reference in the present case the complete information on the state of the two-qubit register is also obtainable.

We also whish to remark that, within the model of trapped electron, other schemes could be exploited, for example by encoding information in other degrees, or by using Schroedinger cat states as well [29]; in fact the latter were shown to be achievable in such systems [21,30].

The next step would be the extension of the above formalism to the case of two or more trapped electrons, in order to investigate real possibilities for quantum registers. One should consider that the realization of a 4-qubit system would be a real advancement because of the possibility of checking error correction strategies. As a final comment we can say that with this simple system we have introduced here, one can implement [31] the Deutsch problem [3, 32] as well.

The authors are grateful for a critical reading of the manuscript by I. Marzoli. This work has been partially supported by INFM (through the 1997 Advanced Research Project "CAT"), by the European Union in the framework of the TMR Network "Microlasers and Cavity QED", by MURST under the "Cofinanziamento 1997" and by the CNR-ICCTI joint programme.

[1] R. Landauer, Phys. Today 44(5), 23 (1991)

[2] R. Feynman, Int. J. Theor. Phys.21, 467, (1982)

[3] D. Deutsch, Proc. R. Soc. London A 400, 97, (1985), ibid. 425, 73, (1989)

[4] D. P. DiVincenzo, Science 270, 255 (1995); S. Lloyd, Sci. Am. (Int. Ed.) 273, 140 (1995); A. Ekert and R. Jozsa, Rev. Mod. Phys. 68, 733 (1995).

[5] D. P. DiVincenzo, Phys. Rev. A 51, 1015 (1995); A. Barenco et al., ibid., 52, 3457 (1995); S. Lloyd, Phys. Rev. Lett. 75, 346 (1995).

[6] R. P. Feynman, Opt. News 11, 11 (1985).

[7] A. Barenco, D. Deutsch, A. Eckert and R. Jozsa, Phys. Rev. Lett. 74, 4083 (1995).

[8] J. I. Cirac and P. Zoller, Phys. Rev. Lett. 74, 4091 (1995).

[9] D. Deutsch and A. Ekert, Phys. World 11, 47 (1998)

[10] Q.A. Turchette, C.S. Wood, B.E. King, C.J. Myatt, D. Leibfried, W. M. Itano, C. Monroe, and D. J. Wineland, Phys. Rev. Letts. 81, 3631 (1998).

[11] Q. A. Turchette, C. J. Hood, W. Lange, H. Mabuchi, and H. J. Kimble, Phys. Rev. Lett. 75, 4710 (1995).

[12] P. Domokos, J. M. Raimond, M. Brune and S. Haroche, Phys. Rev. A 52, 3554 (1995).

[13] D. G. Cory et al. Phys. Rev. Lett. 81, 2152 (1998).

[14] R. Shack and C. M. Caves, e-print: quant-ph/9903101.

[15] F. M. Penning, Physica (Amsterdam) 3, 873 (1936).

[16] R. S. Van Dyck, Jr., P. B. Schwinberg and H. Dehemelt, in Atomic Physics 9, edited by R. S. Van Dyck, Jr. and E. N. Fortson (World Scientific, Singapore, 1984); R. S. Van Dyck, Jr., P. B. Schwinberg and H. Dehemelt, Phys. Rev. Lett. 59, 26 (1987); R. S. Van Dyck, Jr., in Quantum Electrodynamics, edited by T. Kinoshita (World Scientific, Singapore, 1990).

[17] W. H. Zurek, Phys. Today 44(10), 36 (1991); R. Landauer, in Proc. Drexel Fourth Symposium on Quantum Nonintegrability-Quantum Classical Correspondence, D. H. Feng and B. L. Hu Eds. (International Press, Boston, 1996); W. G. Unruh, Phys. Rev. A 51, 992 (1995).

[18] L. S. Brown and G. Gabrielse, Rev. Mod. Phys. 58, 233 (1986).

[19] J. D. Jackson, Classical Electrodynamics, (Wiley, New York, 1975). 
[20] G. Gabrielse and J. Tan, in Cavity Quantum Electrodynamics, edited by P. R. Berman, (Academic Press, San Diego CA, 1994), p.267.

[21] A. M. Martins, S. Mancini and P. Tombesi, Phys. Rev. A 58, 3813 (1998).

[22] C. Monroe, D. Leibfried, B. E. King, D. M. Meekhof, W. M. Itano and D. J. Wineland, Phys. Rev. A 55, R2489 (1997).

[23] S. Mancini and P. Tombesi, Phys. Rev. A 56, 3060 (1997).

[24] G. Gabrielse and H. G. Dehmelt, Phys. Rev. Lett. 55, 67 (1985).

[25] G. Gabrielse, H. G. Dehmelt and W. Kells, Phys. Rev. Lett. 54, 537 (1985).

[26] G. J. Milburn, Phys. Rev A 44, 5401 (1991).

[27] R. Bonifacio, Nuovo Cimento B 114, 473 (1999), eprint: quant-ph/ 9901063.

[28] C. Monroe, D. M. Meekhof, B. E. King, W. M. Itano and D. J. Wineland, Phys. Rev. Lett. 75, 4714 (1995).

[29] P. T. Cochrane, G. J. Milburn and W. J. Munro, eprint: quant-ph/9809037.

[30] S. Mancini and P. Tombesi, Phys. Rev. A 56, R1679 (1997).

[31] I. Marzoli and P. Tombesi (unpublished).

[32] A. Ekert and C. Macchiavello in Unconventional Models of Computation, edited by C.S. Claude, J. Casti and M.J. Dinnen, pp. 19-44. Springer Series in Discrete Mathematics and Theoretical Computer Science (Springer, Singapore, 1998). 